\title{
DETERMINANTES ECONÓMICOS DE LAS REMUNERACIONES EN LAS MANUFACTURAS MEXICANAS
}

\author{
Carlos Guerrero de Lizardi*
}

(Recibido: Diciembre 2010 / Aprobado: Febrero 2011)

\begin{abstract}
"A statistical model constitutes a set of probabilistic assumptions related to random variables giving rise to the data chosen by a theory. Such a model is said to be statistically adequate when the underlying assumptions are tested and not rejected by the data in question."
\end{abstract}

Aris Spanos (1989, p. 151)

\section{RESUMEN}

Para explorar los determinantes económicos de las remuneraciones en las manufacturas mexicanas se adaptó un modelo de competencia imperfecta para economías pequeñas y abiertas, y se realizó un análisis moderno de series de tiempo. Los hallazgos indican que la variable en cuestión juega el papel de residuo en un sistema compuesto por el margen de ganancia, la productividad del trabajo y la competitividad, y que el impacto de las segundas sobre la primera es distinto si se opera en una economía cerrada o en una abierta. La variable clave es la productividad del trabajo por su efecto positivo (en una economía cerrada) y más positivo

* Tecnológico de Monterrey, Campus Ciudad de México. Correo electrónico: <carlos.guerrero. de.lizardi@itesm.mx>. 
Panorama ECONÓMICO, vol. VI, núm. 12, enero-junio de 2011

(en una economía abierta) sobre las remuneraciones, y por su asociación directa con la competitividad.

Palabras claves: Remuneraciones, productividad del trabajo, competitividad Clasificación JEL: E24, E12, C32

\begin{abstract}
In order to explore the economic determinants of the real salaries in Mexican manufacturers a model of imperfect competition for small and open economies was used and a modern time series analysis was performed. The findings show that the variable in question plays a residual role in a system composed, additionally, by profit margin, labor productivity, and competitiveness. The reliable empirical evidence also shows that the impact of the other factors on the first factor is distinct if it operates in a closed economy as compared to its operation in an open economy. The key variable is labor productivity, for both its positive effects (in a closed economy), and its even greater positive effects (in an open economy), on salaries, and for its direct association with competitiveness.

Keywords: Wages, labor productivity, competitiveness

JEL Classification: E24, E12, C32
\end{abstract}

\title{
1. INTRODUCCIÓN
}

Nuestro propósito es examinar los determinantes económicos de las remuneraciones en las manufacturas mexicanas. Para llevarlo a cabo realizamos un pormenorizado análisis de series de tiempo con base a la Encuesta Industrial Mensual Ampliada (EIMA-SCIAN), del Instituto Nacional de Estadística y Geografía (INEGI), cuyo horizonte temporal abarca de enero de 2005 a octubre de 2010.

A continuación revisamos las siguientes cuatro secciones. En la segunda exponemos brevemente nuestro marco teórico de referencia. Para dar los primeros pasos en la comprensión de las variables en juego, en la tercera sección realizamos 
un análisis estadístico preliminar. Con la intención de obtener la mayor cantidad de "evidencia empírica confiable" (Spanos, 1999), en la cuarta llevamos a cabo un análisis de series de tiempo siguiendo el principio de "test, test, and test" (Hendry, 1995) según el "estado del arte" actual. Para cerrar el documento anotamos algunas reflexiones finales.

\section{MODELO TEÓRICO}

El siguiente conjunto de ecuaciones corresponde a un modelo de competencia imperfecta, esto es, a una economía en la que algunas empresas, digamos las dominantes en cada mercado (industria), son "hacedoras de precios" en el sentido en que fijan su propio margen de ganancia y el resto más bien "sigue los precios". Iniciamos con una economía cerrada. El costo laboral unitario equivale al costo salarial por unidad de producto:

$$
\frac{W E}{Y}=\frac{W}{L P}
$$

Donde la productividad del trabajo (LP) se define como:

$$
L P=\frac{Y}{E}
$$

Siguiendo la regla del margen de ganancia $(\mu)$, el precio se determina así:

$$
P=(1+\mu)\left(\frac{W}{L P}\right)
$$

Dicho brevemente, $\mu$ depende del poder de mercado de las empresas líderes, y se supone relativamente estable. Otra manera de expresar (3) es:

$$
P=\left(\frac{1}{1-m}\right)\left(\frac{W}{L P}\right)
$$

Donde: 
Panorama ECONÓMICO, vol. VI, núm. 12, enero-junio de 2011

$$
m=\frac{\mu}{(1+\mu)}
$$

Reescribiendo (4) tenemos:

$$
P=m P+\frac{W}{L P}
$$

Esto es, el precio se descompone en dos partes. La primera corresponde al margen de ganancia unitario, y la segunda al costo laboral unitario que, como vimos, depende inversamente de la productividad del trabajo. Si dividimos entre el precio y multiplicamos por la productividad aparente del trabajo llegamos a:

$$
L P=m L P+\frac{W}{P}
$$

La ecuación anterior pone de relieve la "disputa" sobre el producto generado por trabajador, o puesto con otras palabras, su igualdad con la suma del beneficio real obtenido por trabajador y el salario real por trabajador, lo que representa el límite fijado por la productividad del trabajo a las aspiraciones económicas de los participantes en juego. Dadas las variables contenidas en la ecuación anterior, a saber, el margen de ganancia, la productividad del trabajo y el salario nominal, la fijación del precio implica un determinado nivel de salario real:

$$
w=\frac{W}{P}=L P(1-m)
$$

La Gráfica 1 ilustra lo dicho.

Tomando logaritmos naturales de la ecuación (4) y diferenciando respecto al tiempo obtenemos las siguientes expresiones:

$$
\begin{gathered}
\log (P)=\log \left(\frac{1}{1-m}\right)+\log (W)-\log (L P) \\
\frac{\partial \log (P)}{\partial t}=\frac{\partial \log (W)}{\partial t}-\frac{\partial \log (L P)}{\partial t}
\end{gathered}
$$




\section{GRÁFICA 1}

\section{Determinación del salario real en una economía cerrada}

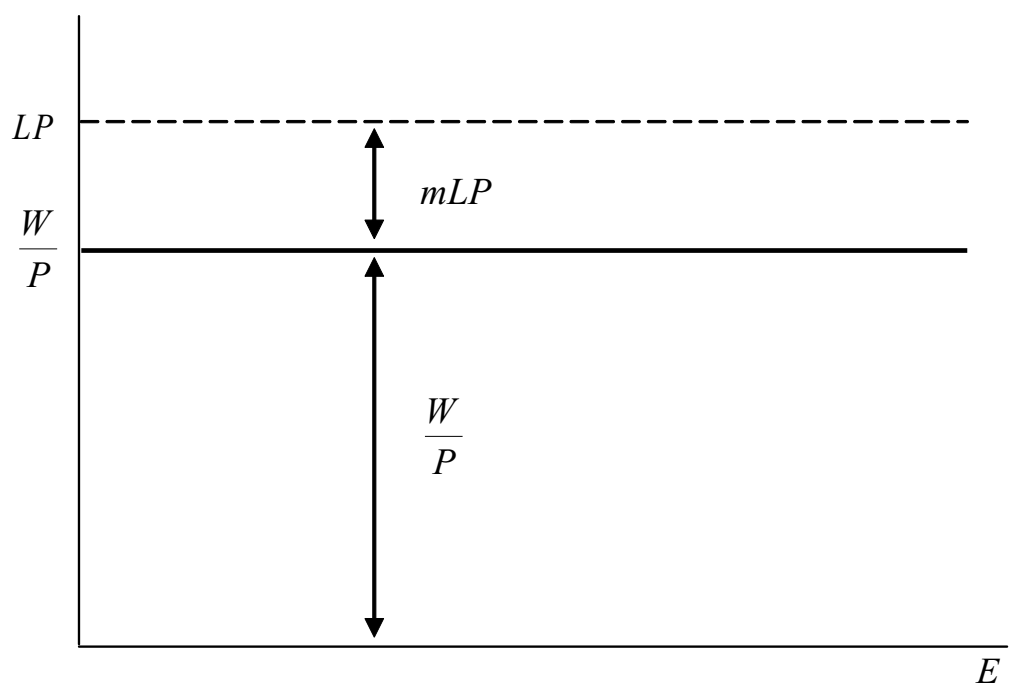

Por su parte, la tasa de crecimiento del salario nominal evoluciona según la siguiente ecuación:

$$
\frac{\partial \log (W)}{\partial t}=\frac{\partial \log \left(P^{E}\right)}{\partial t}+\frac{\partial \log (L P)}{\partial t}+\frac{\partial \log \left(\frac{\text { Empleo Formal }}{\text { Empleo Total }}\right)}{\partial t}
$$

Donde el supraíndice $E$ representa la expectativa. Según el último término de la ecuación, la capacidad de negociación del trabajador depende de la situación del mercado de trabajo. Sustituyendo en (10) y cancelando, obtenemos:

$$
\frac{\partial \log (P)}{\partial t}=\frac{\partial \log \left(P^{E}\right)}{\partial t}+\frac{\partial \log \left(\frac{\text { Empleo Formal }}{\text { Empleo Total }}\right)}{\partial t}
$$


La ecuación anterior sugiere que la formalización del mercado de trabajo presionaría el precio al alza y su informalización a la baja. En este sentido, la economía subterránea representa un mecanismo espurio de contención de precios y salarios.

En una economía abierta tenemos que reescribir (6) de la siguiente manera:

$$
P=m P+\frac{W}{L P}+\frac{P * e}{M P}
$$

El tercer término representa el costo unitario de los insumos importados en moneda local, es decir, $\left(\frac{1}{M P}\right)$ representa el costo, $M P$ la productividad y, $\left(P^{*}\right.$ e) la traducción del precio externo utilizando el tipo de cambio nominal. Dividiendo por el precio y utilizando la definición más común del tipo de cambio real $\left(\theta=\frac{P^{*} e}{P}\right)$, esto es, un incremento en el parámetro de teta, implica dar más unidades monetarias locales por un dólar o, lo que es equivalente, ganar competitividad y, para el caso contrario podemos reescribir (13) de sugerentes maneras:

$$
\begin{gathered}
1=m+\frac{w}{L P}+\frac{\theta}{M P} \\
L P=m L P+w+\theta\left(\frac{L P}{M P}\right) \\
w=L P-m L P-\theta\left(\frac{L P}{M P}\right)
\end{gathered}
$$

La ecuación (15) revela que, en la "disputa" sobre el producto por trabajador en una economía abierta surge un tercer participante aproximado por el costo unitario real de los insumos importados; y, que la satisfacción de las exigencias de los participantes tiene como variable clave, o límite objetivo, la productividad del trabajo. Por su parte, la expresión (16) coloca al salario real como la variable 
residuo, o lo que es equivalente, sugiere una relación inversa entre el salario real y la competitividad de la economía, dados los valores del resto de variables en juego. Sólo si suponemos una productividad del trabajo estancada y un margen de ganancia constante, (16) plantea una disyuntiva entre una política salarial que busque mejorar la situación del trabajo y un objetivo de balanza de pagos. La Gráfica 2 resume el argumento planteado:

\section{GrÁFICA 2}

\section{Determinantes del salario real en una economía abierta}

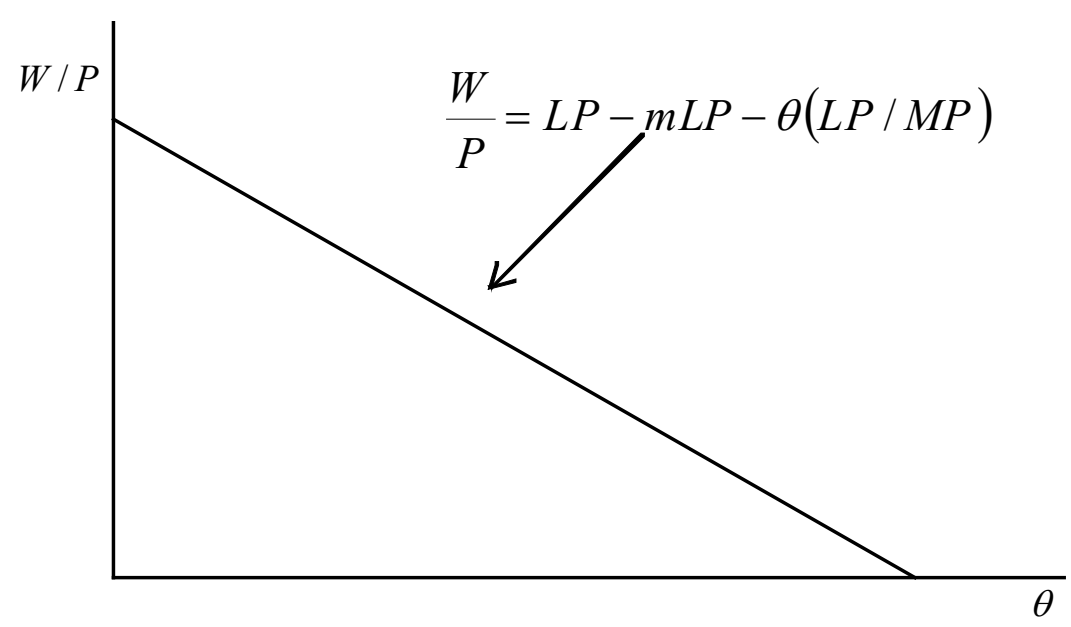

El marco teórico expuesto para el caso de una economía cerrada, específicamente la ecuación (10), inspiró el trabajo econométrico realizado por Catalán (2006). Por nuestra parte utilizaremos la siguiente especificación:

$$
\frac{\partial \log (\mathrm{W})}{\partial \mathrm{t}}-\frac{\partial \log (\mathrm{P})}{\partial \mathrm{t}}=\frac{\partial \log (\mathrm{LP})}{\partial \mathrm{t}}-\frac{\partial \log \left(\frac{\mathrm{P} * \mathrm{e}}{\mathrm{P}}\right)}{\partial \mathrm{t}}
$$

A continuación inspeccionamos estadísticamente la base de datos disponible para llevar a cabo nuestro estudio. 
Panorama ECONÓMICO, vol. VI, núm. 12, enero-junio de 2011

\section{ANÁLISIS ESTADÍSTICO PRELIMINAR}

En la Gráfica 3 observamos las tasas de crecimiento suavizadas (SM) del índice de volumen físico de la producción manufacturera (volumen, 2003=100), del índice global de actividad económica (IGAE, 2003=100) y del índice de la producción industrial de nuestro principal socio económico (IPIUS, 2007=100).

\section{GrÁFICA 3}

Tasas de crecimiento suavizadas de volumen

(IGAE eje izquierdo; IPIUS eje derecho)

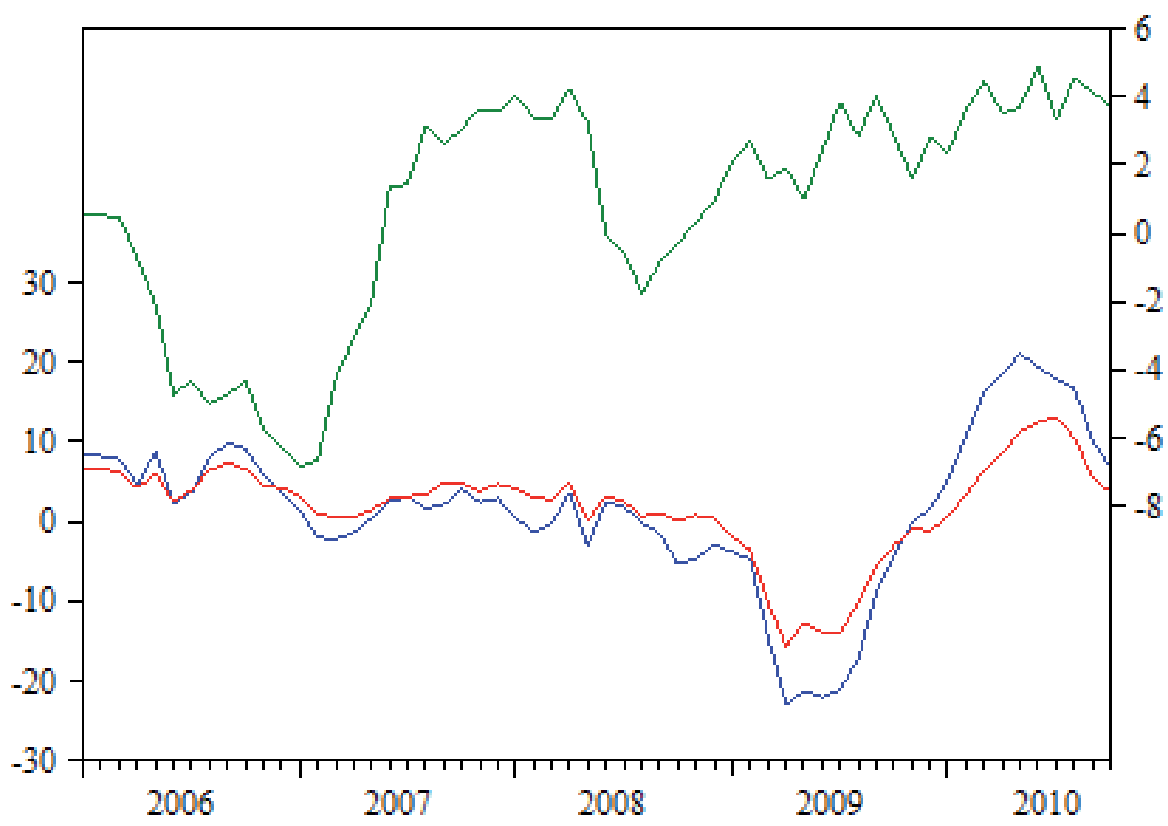

DVOLUMENSM - DIGAESM DIPIUSSM

Fuente: elaboración propia con base en la BIE del INEGI, y la FED. 
Como esperábamos, las variables referidas a la economía mexicana se acompañan en el tiempo (su coeficiente de correlación contemporáneo es de casi uno) y la producción manufacturera acusa mucha mayor volatilidad que el indicador global (su coeficiente de variabilidad es casi el doble). Por otro lado, la Gráfica 3 no proporciona una pista clara respecto a la existencia de un estímulo de demanda de la producción estadounidense hacia la mexicana. Al respecto, las pruebas de no causalidad en el sentido de Granger con distinto número de rezagos apuntaron a que, en efecto, la producción manufacturera mexicana es parcialmente estimulada por la americana y no lo contrario. Para no perder la señal de largo plazo, cabe advertir que, las pruebas se realizaron utilizando los niveles de las variables, lo que abre la posibilidad de que se trate de un resultado espurio.

La Gráfica 4 revela que la producción manufacturera mexicana, o volumen, y el uso del acervo de capital están correlacionados, ambos, positivamente con el número de horas trabajadas. Se trata de una buena noticia por sus implicaciones en términos de la asociación positiva entre la actividad económica y la generación de empleos, y sobre las decisiones de inversión que van más allá del corto plazo. Cabe destacar que, desafortunadamente, la EIMA-SCIAN no proporciona información respecto al acervo de capital propiamente dicho, por lo que no es viable explorar, econométricamente, las características de la función de producción del sector analizado. ${ }^{1}$

Antes de realizar un análisis gráfico conjunto presentamos en la Gráfica 5 los niveles de dos de las tres variables claves para el estudio propuesto, el índice de las remuneraciones medias reales por hora hombre trabajada $(2003=100) \mathrm{y}$ la productividad real por hora hombre trabajada $(2003=100)$, ambas con ajuste (_SA) utilizando el método conocido como Census X12 y sin ajuste por estacionalidad. Es visible la presencia de una tendencia con pendiente positiva en ambos casos.

\footnotetext{
${ }^{1}$ La única fuente de información directa (observada) sobre el acervo de capital la encontramos en los Censos Económicos. Desafortunadamente, según Guerrero (2009) existe un problema de valoración nominal y real del mismo.
} 


\section{GráFICA 4}

Asociación positiva entre las horas trabajadas y volumen y uso de capital
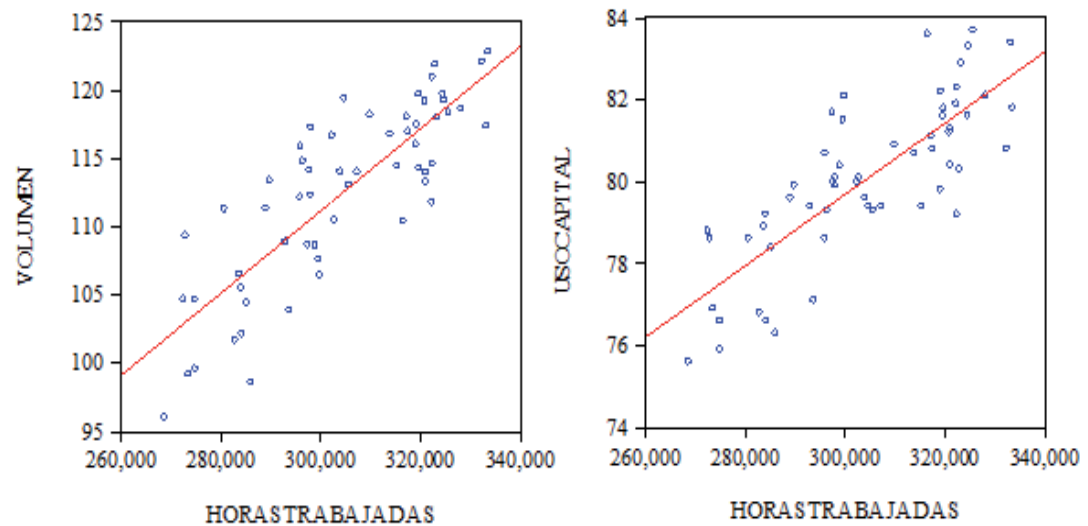

Fuente: elaboración propia con base en la BIE del INEGI.

GráFICA 5

Remuneraciones y productividad en niveles con/sin estacionalidad
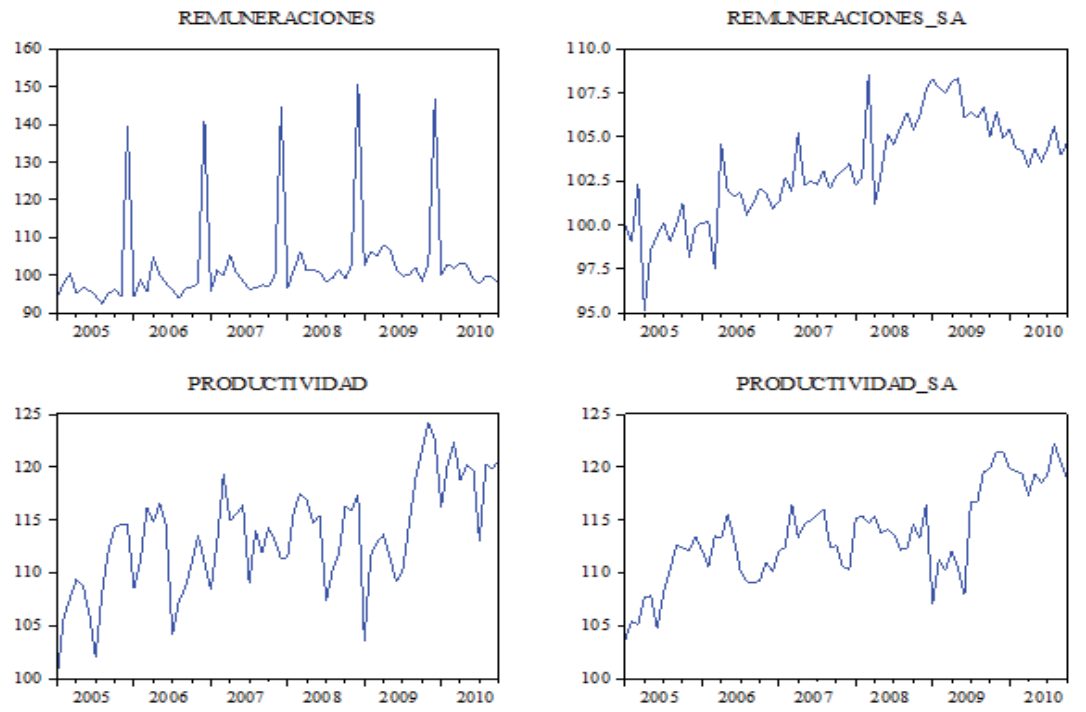

Fuente: elaboración propia con base en la BIE del INEGI. 
El propósito de la Gráfica 6 es recordarnos que, por lo menos en las manufacturas mexicanas, en la teoría y en la práctica detrás de las remuneraciones reales encontramos la productividad aparente del trabajo. Para no distorsionar la escala de los ejes dejamos de lado la información correspondiente a los meses de diciembre. El coeficiente de correlación contemporáneo para este par de variables ascendió a 0.43 .

\section{GrÁFICA 6}

\section{Asociación positiva entre las remuneraciones y la productividad del trabajo}

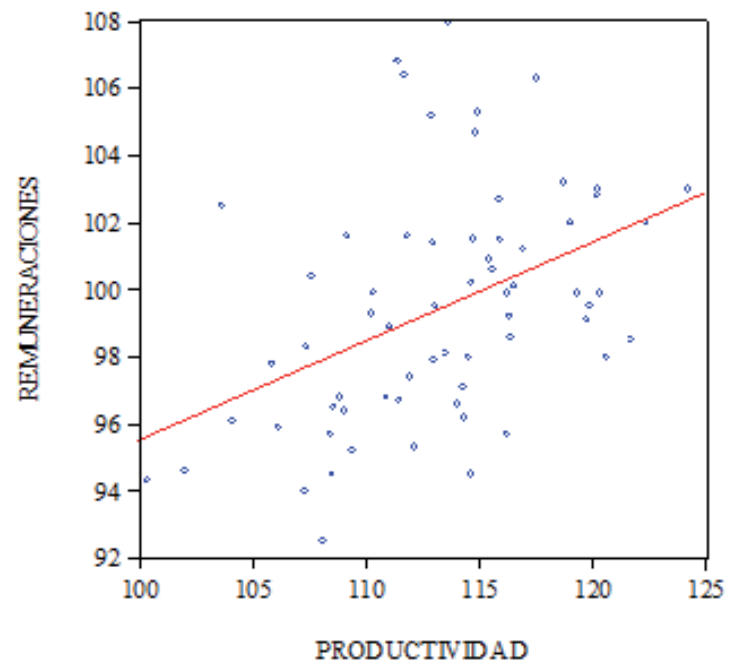

Fuente: elaboración propia con base en la BIE del INEGI.

Cerramos la presente sección con una gráfica que contiene el cociente de los índices de precios de las exportaciones e importaciones, ajustados por el tipo de cambio $(1980=100)$, una medida de competitividad derivada de la comparación de los costos laborales unitarios entre países $(2003=100)$ y el tipo de cambio nominal (pesos por dólar estadounidense). La competitividad constituye nuestra tercera variable clave. 


\section{GrÁFICA 7}

Dos mediciones de la competitividad de las manufacturas mexicanas y el tipo de cambio nominal
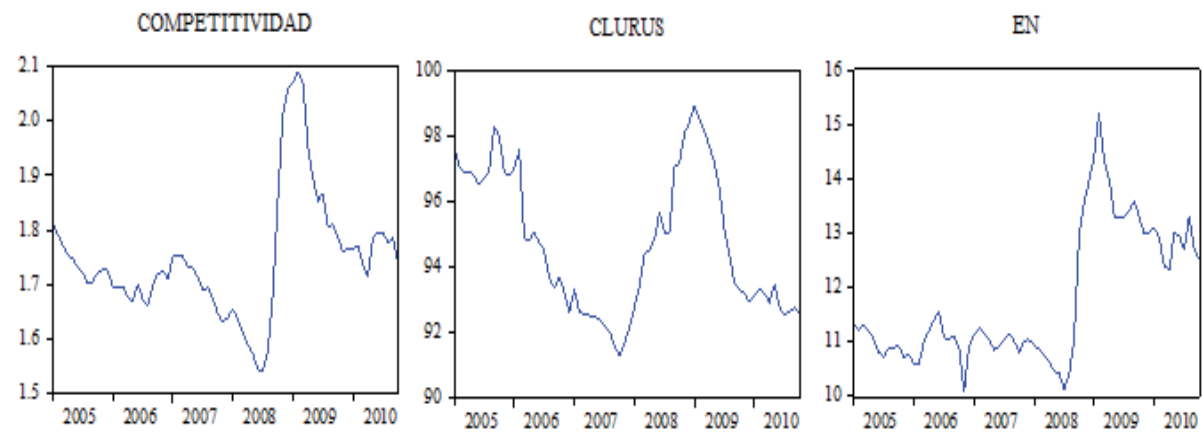

Fuente: elaboración propia con base en la BIE del INEGI y "Estadísticas" de BANXICO.

Si bien los índices de precios de las exportaciones e importaciones son crecientes en el tiempo, parece que su cociente es más bien una variable "aplanada" con un quiebre a finales del 2008. La competitividad en términos de precios parece dominada por la evolución del tipo de cambio nominal, mientras que la definida por los costos laborales unitarios relativos parece más bien adelantar la volatilidad de la relación peso-dólar (López y Guerrero, 1998).

\section{ANÁLISIS DE SERIES DE TIEMPO}

Determinar empíricamente el orden de integración de una variable es absolutamente relevante. No sólo porque la existencia de una tendencia estocástica nos revela el comportamiento de largo plazo de la variable en cuestión. Sobre todo, porque el análisis de series de tiempo nos ayuda a comprender mejor su sentido económico. En nuestro caso, revisaremos cuidadosamente las remuneraciones reales, la productividad del trabajo y la competitividad de las manufacturas. Si bien seguiremos la estrategia propuesta por Dolado, Jenkinson y Sosvilla-Rivero 
(1990) para determinar el orden de integración, el amable lector descubrirá que la reflexión económica servirá de mucha ayuda para llevar a buen puerto, esperamos, nuestro análisis.

Algunas pistas. Desde una perspectiva de largo plazo esperamos que el trabajo sea, con el transcurrir del tiempo, más productivo y, aún más, que presente pequeños y grandes saltos derivados de la incorporación de nuevas tecnologías. Si el mercado de trabajo y los arreglos institucionales funcionan adecuadamente entonces los incrementos de la productividad del trabajo se reflejarán en mejores salarios reales. Por su parte, se espera que la combinación de dos variables integradas, los precios de las exportaciones y de las importaciones, genere una variable estacionaria, en nuestro caso la competitividad internacional. Aquí el tipo de cambio nominal juega el papel de operador. Como se trata de la relación peso-dólar esperamos significativas depreciaciones y ralentizadas apreciaciones. Sorprendentemente, la Gráfica 7 nos sugirió cómo una variable que se espera estacionaria puede seguir a una variable no estacionaria.

La base de datos es, en términos estadísticos, suficientemente amplia pues, disponemos de 70 observaciones, sin embargo en términos económicos abarca apenas seis años. La determinación del orden de integración de las variables en cuestión estará acotado, entonces, a un reducido horizonte temporal, o dicho con otras palabras, obtendremos una respuesta limitadamente empírica. No puede ser de otra manera.

La estacionalidad es un componente relevante de las remuneraciones reales, sobre todo en el mes de diciembre. En este sentido, aplicamos repetidamente las pruebas Dickey-Fuller aumentada y Phillips-Perron, las pruebas más influyentes de la literatura de raíces unitarias (Bierens, 2003, p. 610), con las variables “enteras" y desestacionalizadas. ${ }^{2}$ Verificamos tanto la significancia estadística de los términos determinísticos (constante y tendencia) como los rezagos de la propia variable, hasta doce, si aplican. Asimismo verificamos que los residuales de las

\footnotetext{
2 Para la hipótesis de componentes no observables o subyacentes una serie de tiempo está hecha de cuatro partes: la tendencia, el ciclo, la estacionalidad, y el ruido.
} 
pruebas se comportaran como "ruido blanco". De acuerdo con nuestro análisis puede aceptarse que las tres variables que servirán para especificar un modelo VAR según la ecuación (17), se comportan de manera no estacionaria, con un orden de integración I(1), esto es, se trata de variables con memoria larga, lo que implica, por ejemplo, que cualquier choque recibido en algún momento no se diluya sino que permanezca en el tiempo.

Inicialmente para explorar el efecto de la productividad del trabajo sobre las remuneraciones reales en una economía cerrada impusimos una restricción radical, a saber, dejamos fuera del modelo VAR la competitividad. Evidentemente se trató de un ejercicio limitadamente contrafáctico. Posteriormente re-especificamos según la ecuación (17). Ponderando los resultados de las muchas pruebas de información (hasta 12 rezagos) sobre la condición de estabilidad del modelo estimado y de la incorrecta especificación (autocorrelación, normalidad y heteroscedasticidad), seleccionamos un modelo VAR con, curiosamente, seis rezagos. Evidentemente utilizamos los logaritmos naturales de las variables en juego. Los resultados de las pruebas de Johansen arrojaron los siguientes vectores de cointegración:

TABLA 1

Vectores de cointegración

\begin{tabular}{|c|c|c|}
\hline LOG(REMUNERACIONES) & LOG(PRODUCTIVIDAD) & LOG(COMPETITIVIDAD) \\
\hline 1.000000 & -0.947202 & \\
\hline 1.000000 & -1.001265 & 0.593721 \\
\hline
\end{tabular}

Los coeficientes contenidos en la Tabla 1 representan las elasticidades entre las variables y deben leerse con el signo opuesto. Parece que existe una pequeña diferencia del impacto de la productividad del trabajo sobre las remuneraciones reales si suponemos una economía cerrada o una abierta. Destacadamente obtuvimos una elasticidad prácticamente unitaria, esto es, cada punto porcentual de incremento de la productividad del trabajo se reflejará, ceteris paribus, en un incremento similar de las remuneraciones reales. Desde el punto de vista estric- 
tamente económico, lo anterior refleja el mejor arreglo posible entre los trabajadores y los empresarios del sector analizado. Por su parte la elasticidad ligada a la competividad resultó negativa, como esperábamos. Pero el efecto dominante está claramente en la productividad del trabajo. A propósito, si reordenamos las variables descubrimos que la elasticidad que vincula a la competitividad y a la productividad del trabajo es positiva. Y las pruebas de exogeneidad indicaron que es correcto identificar las remuneraciones reales como la variable residual del sistema propuesto.

\section{CONCLUSIONES}

Para explorar los determinantes económicos de las remuneraciones reales en las manufacturas mexicanas, se adaptó un modelo de competencia imperfecta para economías pequeñas y abiertas y se realizó un moderno análisis de series de tiempo. Descubrimos que las remuneraciones reales juegan el papel de residuo en un sistema compuesto adicionalmente por otras tres, el margen de ganancia, la productividad del trabajo y la competitividad de la economía, y que el impacto de las segundas sobre la primera es distinto si se opera en una economía cerrada y en una abierta. Al respecto subrayemos que la variable clave es la productividad del trabajo por su efecto positivo (en una economía cerrada) y más positivo (en una economía abierta) sobre las remuneraciones, y por su asociación directa con la competitividad.

Para terminar explicitemos algunas tareas pendientes: la aplicación de la especificación propuesta a otras mediciones del ingreso de los trabajadores y a las manufacturas de manera desagregada como un mecanismo de validación de la misma; y, la adición de una variable que capte no sólo el ciclo económico sino el impacto de la economía informal sobre el sistema propuesto. Asimismo, hace falta analizar con detalle la asociación entre la productividad del trabajo y la competitividad macroeconómica internacional, y profundizar nuestra comprensión del impacto diferencial de la productividad del trabajo sobre las remuneraciones bajo funcionamientos económicos cerrados y abiertos. 
Panorama ECONÓMICO, vol. VI, núm. 12, enero-junio de 2011

\section{BIBLIOGRAFÍA}

Bierens, H. J. (2003), "Unit roots", A Companion to Theoretical Econometrics, editado por Badi H. Baltagi, Blackwell Publishing.

Carlin, W. y D. Soskice (1990), Macroeconomics and the Wage Bargain, Oxford University Press.

Catalán, H. (2006), "Productividad, precios y salarios en las economías de Centroamérica y la República Dominicana: un análisis de modelo VAR”, documento de trabajo, CEPAL.

Dolado, J., T. Jenkinson, y S. Sosvilla-Rivero (1990), "Cointegration and unit roots", Journal of Economic Surveys, 4: 3, pp. 249-73.

Guerrero, C. (2009), "Contribution of the information and communication technology sector to Mexican Economic growth from 1999 to 2003", EconoQuantum, 6: 1, pp. 11-30.

Hendry, D. F. (1995), Dynamic Econometrics, Oxford University Press.

Hendry, D. F. (2000), Econometrics: Alchemy or Science?, primera edición en 1993, Oxford University Press.

López, J. y C. Guerrero (1998), “Crisis externa y competitividad de la economía mexicana", El Trimestre Económico, LXV (4): 260, pp. 582-98.

Spanos, A. (1989), "Early empirical findings on the consumption function, stylized facts or fiction: a retrospective view", Oxford Economic Papers, 41:1, pp. $150-69$.

Spanos, A. (1999), Probability Theory and Statistical Inference: Econometric Modeling with Observational Data, Cambridge University Press. 